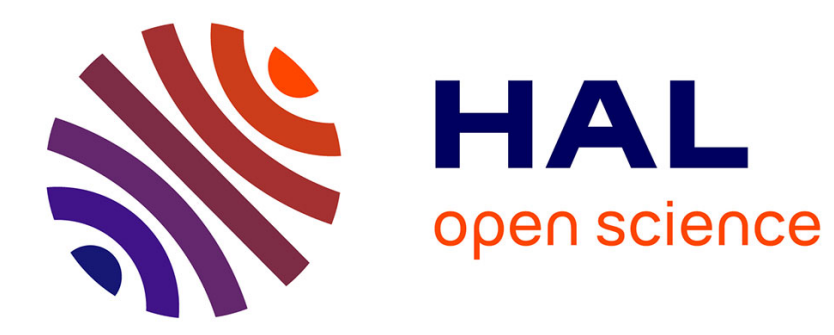

\title{
Sur une locution abandonnée par La Fontaine: fait à fait que
}

\author{
Takeshi Matsumura
}

\section{To cite this version:}

Takeshi Matsumura. Sur une locution abandonnée par La Fontaine: fait à fait que. FRACAS, 2017, 59, pp.1-10. halshs-01531852

\section{HAL Id: halshs-01531852 \\ https://shs.hal.science/halshs-01531852}

Submitted on 2 Jun 2017

HAL is a multi-disciplinary open access archive for the deposit and dissemination of scientific research documents, whether they are published or not. The documents may come from teaching and research institutions in France or abroad, or from public or private research centers.
L'archive ouverte pluridisciplinaire HAL, est destinée au dépôt et à la diffusion de documents scientifiques de niveau recherche, publiés ou non, émanant des établissements d'enseignement et de recherche français ou étrangers, des laboratoires publics ou privés. 


\section{F R A C A S}

numéro 59

le 29 mai 2017

Groupe de recherche

sur la langue et la littérature françaises

du centre et d'ailleurs

(Tokyo)

contact : revuefracas2014@gmail.com 
Sur une locution abandonnée par La Fontaine : fait à fait que

Takeshi MATSUMURA

Dans sa première version parue dans Fables nouvelles, et autres poësies ${ }^{1}$, la Fable Le Coche et la Mouche contient au vers 11 une locution remarquable ${ }^{2}$. Citons la phrase qui la contient.

Une Mouche survient, \& des chevaux s'approche ;

Prétend les animer par son bourdonnement ;

Pique l'un, pique l'autre, \& pense à tout moment

Qu'elle fait aller la machine,

S'assied sur le timon, sur le nez du Cocher :

Fait-à-fait que le char chemine,

Et qu'elle voit les gens marcher,

Elle s'en attribuë uniquement la gloire ;

Va, vient, fait l'empressée ; il semble que ce soit

Un Sergent de bataille allant en chaque endroit

Faire avancer ses gens, \& hâter la victoire. (p. 5)

Le vers 11 est modifié plus tard et l'on lit Aussitôt que $e^{3}$ le char chemine dans l'édition procurée par Jean-Pierre Collinet ${ }^{4}$, qui se fonde sur l'imprimé de 1692. L'éditeur annote de la manière suivante l'expression abandonnée :

Le texte des Fables nouvelles, en 1671, portait ici «Fait à fait», que Furetière définit par «à mesure que », locution toujours vivante en Champagne, veut bien me signaler M. Raymond Josse, à qui nous devons tant de découvertes sur la biographie du fabuliste, et notamment sur la partie champenoise de sa vie ${ }^{5}$.

\footnotetext{
1 Paris, Denys Thierry, 1671 ; la citation est faite d'après l'exemplaire de la Bibliothèque de l'Arsenal, 8-BL-16867.

2 Qui n'est pourtant pas étudiée par Arnulf Stefenelli dans son ouvrage Lexikalische Archaismen in den Fabeln von La Fontaine, Passau, Andreas-Haller-Verlag, 1987.

${ }^{3}$ La locution fait à fait que ne signifie pas « aussitôt que », voir pourtant plus loin la note 26, remarque sur Frédéric Godefroy.

${ }^{4}$ La Fontaine, Euvres complètes, t. 1, Fables, contes et nouvelles, Édition établie, présentée et annotée par Jean-Pierre Collinet, Paris, Gallimard, 1991, Bibliothèque de la Pléiade, p. 264.

5 Ibid., p. 1176.
} 
À ma connaissance, c'est l'unique note ${ }^{6}$ de cette édition qui soit consacrée au caractère régional du vocabulaire. On peut regretter d'une part que Jean-Pierre Collinet ait jugé suffisant d'alléguer le nom de Raymond Josse sans préciser davantage sa source d'information et d'autre part qu'il ne se soit pas donné la peine de renvoyer à l'interprétation de ses prédécesseurs, qui ne s'accordait pas tout à fait avec la sienne. Peut-être est-ce cette imprécision ou ce laconisme qui a amené Jean-Charles Darmon et Sabine Gruffat à se contenter dans la note de leur édition ${ }^{7}$ de faire remarquer, en se référant seulement à Antoine Furetière ${ }^{8}$, que la locution de la première publication signifie «à mesure ». Leur prudence serait-elle justifiée ? En passant sous silence un éventuel caractère régional du vocabulaire de La Fontaine, n'aurait-elle pas privé les lecteurs de l'occasion de s'y intéresser?

Pour compléter l'observation de Jean-Pierre Collinet, voyons d'abord si l'érudit castelthéodoricien n'ajoute rien dans un de ses nombreux travaux. Heureusement, dans son ouvrage Jehan de La Fontaine à Château-Thierry vu par un homme de son pays ${ }^{9}$, il examine la lettre de La Fontaine à Jannart du 19 août $1659^{10}$, qui contient la locution fait à fait que. C'est cette occurrence qui le conduit à faire une petite digression. Voici l'alinéa :

Et maintenant, une expression de la lettre va nous permettre une petite digression sur le «parler local». Comme toutes les régions de France, la Champagne de Château-Thierry avait son patois qui, sans être une véritable langue, avait cependant son vocabulaire et ses expressions; étant enfant, Jean avait obligatoirement parlé patois avec les gamins de son âge, et, homme mûr, il lui est arrivé, ses écrits le prouvent, de laisser passer une expression patoisante. Le patois local est un mélange de vieux français (par exemple, on a conservé le mot

\footnotetext{
6 Dans son édition de 1974 (La Fontaine, Fables, Livres I à VII, Paris, Gallimard, collection Poésie, note 2 de la page 227), Jean-Pierre Collinet se bornait à traduire l'expression sans mentionner sa diffusion restreinte. L'intervention de Raymond Josse a eu lieu apparemment entre 1974 et 1991.

7 Voir La Fontaine, Fables, Préface de Jean-Charles Darmon, Dossier et notes par Jean-Charles Darmon et Sabine Gruffat, Paris, Librairie Générale Française, 2002, Les Classiques de Poche, p. 217, note 6.

8 Voir Antoine Furetière, Dictionnaire universel contenant généralement tous les mots françois tant vieux que modernes et les termes de toutes les sciences et des arts, La Haye et Rotterdam, 1690, 3 vol., t. 2, p. 12a, s.v. fait à fait : «adv. A mesure, à proportion. J'ay promis de payer mon Maçon fait à fait que mon bâtiment s'avancera. » (c'est l'auteur qui souligne).

9 Société historique et archéologique de Château-Thierry, Maison Jean de La Fontaine, Château-Thierry, 1987.

10 Lettre datée du 19 août 1658 par Pierre Clarac dans son édition ; voir La Fontaine, CEuvres complètes, t. 2, Euvres diverses, Édition établie et annotée par Pierre Clarac, Paris, Gallimard, 1958, Bibliothèque de la Pléiade, p. 484 : «Le mécompte vient aussi de ce que je n'imputais pas les sommes données sur les arrérages précédents fait à fait qu'elles ont été données ». En s'appuyant sur une observation de Jean-Pierre Collinet, Raymond Josse la date de 1659.
} 
« poison » au féminin, « de la poison »), d'idiome local et de français déformé, et quand on veut s'exprimer en français pur, il arrive qu'on parle un peu patois sans s'en rendre compte, tout comme on croit ne pas avoir d'accent. Dans la lettre, on lit «fait à fait»; cette locution a disparu, mais le mot «fait» est resté d'un emploi courant dans la région, grâce à deux locutions «par le fait », qui ne veut à peu près rien dire, et est une simple expression de liaison entre deux phrases, et «à fait», qui a un sens bien précis; cette locution apparaît dans la fable «Le coche et la mouche ». «A fait» signifie « complètement», pour insister, on dit « à fait à fait », et quand on veut dire «sans aucune exception » on dit : «A fait à fait à fait ». C'est pourquoi je suis en désaccord complet avec Walckenaer - une fois n'est pas coutume - qui fait de «à fait à fait » une locution picarde ! (p. 161)

Ainsi, l'érudit admet que la locution fait à fait que a disparu dans sa région et il se base sur l'expression à fait (ou à fait à fait, à fait à fait à fait) pour étayer le caractère régional de l'expression employée par La Fontaine. L'explication donnée par un autochtone est certes respectable, mais elle me semble être un peu confuse ${ }^{11}$ et avoir besoin d'être vérifiée d'après différentes sources.

Avant de compléter l'intuition de Raymond Josse, jetons un coup d'œil sur l'hypothèse émise par Charles Athanase Walckenaer et voyons comment elle a été transmise à la postérité ${ }^{12}$. C'est dans une note de son édition des Fables qu'en citant la variante de l'imprimé de 1671, Walckenaer nous apprend que la locution a une aire de diffusion assez restreinte. Citons la note, qui est très courte :

VAR. Dans le recueil de 1671, p. 5 :

Fait à fait que le char chemine.

Cette expression picarde fait à fait signifie à mesure que, pendant que ${ }^{13}$.

Cette observation est si laconique que l'on ne peut pas savoir sur quelle autorité elle se fonde. L'éditeur disposait-il d'un dictionnaire, d'une étude ou d'un témoin pour avancer cette affirmation? On comprend qu'ainsi conçue sans références, elle a heurté

${ }^{11}$ On ne voit pas très bien ce qu'il entend par patois. Est-ce le dialecte ou le français régional ? Ensuite, pour prouver l'existence de la locution fait à fait que, celle de par le fait et d'à fait au sens de « complètement » est-elle pertinente ? Et enfin où Walckenaer parle-t-il de la locution à fait à fait ?

12 À titre de curiosité, on peut signaler que dans son édition des Euvres complètes de La Fontaine (Paris, Baudouin et Sautelet, 1826, p. 47 et 470), Balzac n'a pas commenté notre locution.

${ }_{13}$ Euvres de La Fontaine, Nouvelle édition, revue, mise en ordre, et accompagnée de notes, par C. A. Walckenaer, t. 2, Paris, Lefèvre, 1827, p. 28, note 3. Naturellement il aurait dû dire : «Cette expression picarde fait à fait que signifie... ». 
le sentiment linguistique de Raymond Josse. Cependant, elle a été reprise, avec ou sans renvoi à sa source, par les éditeurs tels qu'Henri Régnier ${ }^{14}$, Georges Couton ${ }^{15}$ et Marc Fumaroli ${ }^{16}$, qui ont tous considéré notre locution comme picarde. Curieusement, l'érudit castelthéodoricien comme les savants éditeurs semblent avoir négligé une autre note de Walckenaer. En effet, en annotant la proposition fait à fait qu'elles ont été données employée dans la lettre de La Fontaine à Jannart, il donnait une explication un peu différente ${ }^{17}$. La voici :

C'est-à-dire, à mesure qu'elles ont été données. Fait à fait est une locution picarde et champenoise que notre poëte avait employée dans la première édition de la fable intitulée le Coche et la Mouche, mais qu'il a depuis fait disparaître :

Fait à fait que le char chemine.

Fables nouvelles et autres poésies, 1671, p. 5.

Cette note a été recopiée textuellement par Louis Moland dans son édition de La Fontaine $^{18}$. Mais ni Walckenaer ni Moland n'ont jugé nécessaire de préciser sur quoi ils se sont appuyés pour affirmer le caractère régional de la locution. Et leur observation a été oubliée dans les éditions et les études ${ }^{19}$ parues à leur suite.

La locution est-elle picarde ? Ou est-elle picarde et champenoise ? Ou encore n'est-elle pas marquée régionalement? Voyons si nos instruments de travail habituels peuvent nous aider à répondre à ces questions. Malheureusement le Dictionnaire de la

14 Voir CEuvres de J. de La Fontaine, Nouvelle édition revue sur les plus anciennes impressions et les autographes et augmentée de variantes, de notices, de notes, d'un lexique des mots et locutions remarquables, de portraits, de fac-simile, etc. par M. Henri Régnier, t. 2, Paris, Hachette, 1884, Les Grands Écrivains de la France, p. 142, note 7 : « [...] c'est, dit Walckenaer, une locution picarde. [...]. » Le Lexique du tome 10 (voir Lexique de la langue de J. de La Fontaine avec une introduction grammaticale par M. Henri Régnier, t. 1, Paris, Hachette, 1892, p. 371) ne fait que renvoyer à cette note.

15 Voir La Fontaine, Fables choisies mises en vers, Introduction, notes et relevé de variantes par Georges Couton, Paris, Garnier, 1962, p. 472, note 3 de la page 189, avec renvoi à Walckenaer.

16 Voir La Fontaine, Fables, Texte présenté et commenté par Marc Fumaroli, Paris, Imprimerie Nationale, 1985, 2 vol., t. 2, p. 331, note 11 de la page 33 : «Aussitôt que : 1671 : "fait à fait que” (loc. picarde.) » sans renvoi à Walckenaer. Cette note reste la même dans la réimpression (La Fontaine, Fables, Édition établie, présentée et annotée par Marc Fumaroli, Paris, Librairie Générale Française, 1995, La Pochothèque, p. 876, note 11 de la page 401) ; l'éditeur n'a apparemment pas pris en considération la note de Jean-Pierre Collinet glissée dans sa publication de 1991.

17 Voir CEuvres complètes de Jean de La Fontaine avec des notes et une nouvelle notice sur sa vie, par M. C. A. Walckenaër, Paris, Didot, 1836, p. 633b, note 2.

18 Voir CEuvres complètes de La Fontaine. Nouvelle édition très-soigneusement revue sur les textes originaux avec un travail de critique et d'érudition, aperçus d'histoire littéraire, vie de l'auteur, notes et commentaires, bibliographie, etc. par M. Louis Moland, t. 7, Paris, Garnier, 1886, p. 297, note 2.

19 Voir par exemple Théodore Lorin, Vocabulaire pour les Euvres de La Fontaine ou explication et définition des mots, locutions, formes grammaticales, etc. employés par La Fontaine et qui ne sont plus usités, Paris, Comon, 1852 et la recension de cet ouvrage, due à Charles Marty Laveaux, «Essai sur la langue de La Fontaine », Bibliothèque de l'École des Chartes, 1853, p. 484-511 ; 1854, p. 58-82. 
langue française d'Émile Littré ${ }^{20}$, le Trésor de la langue française de Paul $\operatorname{Imbs}^{21}$ et la Base historique du vocabulaire français ${ }^{22}$ ignorent tous notre locution. Par contre, le Französisches Etymologisches Wörterbuch de Walther von Wartburg ${ }^{23}$ lui consacre un alinéa de son article factum (t. 3, p. 362a). Citons-le en développant les abréviations et en ajoutant des précisions géographiques entre crochets carrés :

Moyen français a fait que « à mesure que »; français moderne fait à fait que « à mesure que » (XVII ${ }^{\mathrm{e}}$ siècle) ; wallon a fait « au fur et à mesure », namurois Givet [Ardennes] Tourcoing [Nord] Lille fait à fait, rouchi fet à fet, Mons fait à fait (déjà chez Philibert Delmotte ${ }^{24}$ ), Dombras [Meuse] fāt a fāa, lorrain fait à fait $q u e$ «à mesure que » chez Jean-François Michel $^{25}$, Vouthon [Meuse] à fa «id. ; sans choisir ».

D'après ces indications ${ }^{26}$ du FEW, la locution conjonctive fait à fait que est donc attestée en français au XVII ${ }^{\mathrm{e}}$ siècle (qui se réfère sans doute à Furetière et éventuellement à La Fontaine) et en français régional de Lorraine au début du XIX ${ }^{\mathrm{e}}$ siècle. En fait, les dialectes lorrain et liégeois la connaissent aussi si l'on suit le Glossaire abrégé du

20 Paris, Hachette, 1873, 4 vol.

21 Paris, CNRS et Gallimard, 1971-1994, 16 vol.

22 Consultable sur le site suivant : http://www.cnrtl.fr/definition/bhvf/.

23 Bâle, etc., Zbinden, etc., 1922-2002, 25 vol. ; je désigne ce dictionnaire par FEW.

24 Voir Philibert Delmotte, Essai d'un glossaire wallon, Mons, 1907-1909, 2 vol. ; manuscrit achevé en 1812 selon le Complément du FEW, voir Französisches Etymologisches Wörterbuch, Complément, $3^{\mathrm{e}}$ édition publiée par Jean-Paul Chauveau, Yan Greub et Christian Seidl, Strasbourg, Éditions de linguistique et de philologie, 2010, p. 82b.

25 Il s'agit de Jean-François Michel, Dictionnaire des expressions vicieuses usitées dans un grand nombre de départements, et notamment, dans la ci-devant Province de Lorraine, Nancy, 1807, p. 88 : «FAIT-A-FAIT, FUR-A-MESURE ne sont pas français. Ne dites pas, Étendez ces papiers fait-à-fait que vous les tirez de l'eau. Étendez les fur-à-mesure. - Étendez ces papiers à mesure que vous les tirez de l'eau. Étendez-les au fur et à mesure. » On voit qu'il s'agit du français régional de Lorraine plutôt que du dialecte lorrain.

${ }^{26}$ Qui peuvent être complétées facilement. Par exemple, la locution à fait que est attestée non seulement en moyen français mais depuis l'ancien français, chez Philippe Mousket (hennuyer, vers 1243), voir mon Dictionnaire du français médiéval, Paris, Les Belles Lettres, 2015, p. 1492a, s.v. fait ${ }^{l}$. Les attestations de cette locution relevées dans le Dictionnaire de l'ancienne langue française et de tous ses dialectes du IX au XV siècle de Frédéric Godefroy (10 vol., Paris, 1880-1902, t. 3, p. 708a, s.v. fait) avec une définition erronée ("aussitôt que»; le lexicographe aurait-il été trompé par Le Coche et la Mouche ?) sont du nord-est: Gilles de Chin (hennuyer, deuxième quart du XIII siècle ; voir L'Histoire de Gille de Chyn by Gautier de Tournay edited by Edwin B. Place, Evanston et Chicago, Northwestern University, 1941, vers 5195-5196 : «C'a fait que Hanüier venoient A lor volenté lez prendoient »), deux documents messins du milieu du XIV ${ }^{\text {e }}$ siècle, Froissart (picard, vers 1395 ; voir Froissart, Chroniques, Livre I, Le Manuscrit d'Amiens, Bibliothèque municipale $n^{\circ} 486$ édité par George T. Diller, t. 3, Genève Droz, 1992, § 618, p. 225 : «Et à fet qu'il minoient, il estanchonnoient et chil dou fort riens n'en savoient. »), Julien Fossetier (hennuyer, début du XVI siècle). Sans doute la locution est-elle marquée géographiquement. 
patois de la Meuse de Henri-Adolphe Labourasse ${ }^{27}$ et le Dictionnaire liégeois de Jean Haust $^{28}$. Quant au français régional, le Dictionnaire des belgicismes de Michel Francard ${ }^{29}$ comme le Dictionnaire du français régional du Nord-Pas-de-Calais de Fernand Carton et Denise Poulet ${ }^{30}$ l'ignorent, tandis que les répertoires consacrés à la Lorraine, aux Ardennes et à la Champagne qui enregistrent la locution adverbiale fait à fait ne nous disent pas avec netteté si la locution conjonctive fait à fait que est employée ou non ${ }^{31}$. Est-il possible d'en recueillir ailleurs des attestations ? D'après ma petite enquête, on en trouve plusieurs en français régional, depuis le milieu du $\mathrm{XV}^{\mathrm{e}}$ siècle jusqu'au début $\mathrm{du} \mathrm{XX}^{\mathrm{e}}$ siècle, et sauf des témoignages difficiles à localiser ${ }^{32}$, elles se répartissent dans quatre régions : Picardie, Wallonie, Lorraine et Champagne.

27 Voir Henri-Adolphe Labourasse, Glossaire abrégé du patois de la Meuse, notamment de celui des Vouthons, Arcis-sur-Aube, 1887, p. 277 : «*Fa (à), loc. adv., sans choisir. J'pro à fa, je prends tout, sans faire de choix, sans distinction. On ai pris tourtous los conscrits à fa, on a pris tous les conscrits sans en réformer aucun. || *A mesure que.. L'ai mangé los gaufres à fa que j'los faïeul, il a mangé les gaufres à mesure que je les faisais. - C'est une abréviation de la locution fait à fait que, employée par La Fontaine On dit aussi fâ t'a fâ que... «Fait à fait que le char chemine » (Le Coche et la Mouche, recueil de 1671, p. 5).»

${ }_{28}$ Voir Jean Haust, Dictionnaire liégeois, Liège, Vaillant-Carmanne, 1933, p. 265b, s.v. fêt : « a fêt qui, ou fêt a fêt qui, fêt-a fêt qui, fêt-a fêt' qui, à mesure que ».

29 Bruxelles, Duculot, 2010.

30 Paris, Bonneton, 1991.

31 Voir Jean Lanher et Alain Litaize, Dictionnaire du français régional de Lorraine, Paris, Bonneton, 1990, p. 71b qui a une entrée fait-à-fait, adv., «au fur et à mesure, petit à petit », où ils donnent un exemple : «Il faut travailler fait à fait, dans le désordre on n'arrive à rien. ». De son côté, Michel Tamine, Le parler des Ardennes, Paris, Bonneton, 2006, p. 92b, s.v. fait cite des locutions à fait et fait à fait, qu'il traduit par «au fur et à mesure » avec un exemple : «Les moineaux avalent les graines à fait que je les sème. » Dans Le parler de Champagne, Paris, Bonneton, 2009, p. 67a, s.v. fait, le même auteur cite deux locutions : d'une part, à fait, qu'il traduit par «complètement» avec un exemple : «Il [le docteur] déclara que ce ne pouvait être que des coliques de miserere, et expliqua que le pauvre diable avait l'intestin 'bouché ou noué à fait'. » (Braibant, Le Roi dort), et de l'autre, fait à fait, qu'il traduit par « au fur et à mesure »; pour la deuxième locution il ne donne pas d'exemple. Voir aussi Ch. Cornette, «Le Vieux parler de Pavant», Annales de la Société historique et archéologique de Château-Thierry, 1926, p. 101-128, surtout p. 119 : «FAIT A FAIT, au fur et à mesure : J'posions les bottes fait à fait. »

32 Outre celle de Furetière citée plus haut, voir Armorial de France, Angleterre, Ecosse, Allemagne, Italie et autres puissances Composé vers 1450 par Gilles le Bouvier, dit Berry, [...] éd. A. Vallet de Viriville, Paris, Bachelin-Deflorenne, 1866, p. 40 : «Mais pour hoster les debbats, je les ay mises en ce livre ainsi qu'elles m'ont esté baillées et fait à fait que je les ay trouvées. » (c'est moi qui souligne). Je n'ai pu localiser non plus l'Instruction aux comédiens, parue en tête (sans pagination) de la contrefaçon lyonnaise du Sicilien de Molière, voir Le Sicilien Comedie de Monsieur de Molliere, Paris, Nicolas Pepinglé, 1668 : «Il faut observer que le chassis est de couleur blanche, qu'il y a un visage representant l'Actrice, lequel visage est couvert de blanc, lequel s'efface fait à fait que le pinceau touche dessus, \& oste le d. blanc» (c'est moi qui souligne). L'Instruction a été publiée dans Euvres de Molière, Nouvelle édition revue sur les plus anciennes impressions et augmentée de variantes, de notices, de notes, d'un lexique des mots et locutions remarquables, d'un portrait, de fac-simile, etc. par MM. Eugène Despois et Paul Mesnard, t. 6, Paris, Hachette, 1881, Les Grands Écrivains de la France, p. 303-305. Voir aussi la notice de Molière, Euvres complètes, Édition dirigée par Georges Forestier, avec Claude Bourqui, t. 1, Paris, Gallimard, 2010, Bibliothèque de la Pléiade, p. 1506. La troisième attestation difficile à localiser est la Deduction concernant les droits de succession et de substitution de la Serenissime Maison Electorale de Baviere aux Royaumes de Hongrie et de Boheme, Ainsy qu'a l'Archiduché d'Autriche \& Autres Etats en dépendants, Munich, 1741, p. 64 : «la raison en est, qu'aprés la façon, dont il s'étoit expliqué au sujet des Mâles en 
Les exemples picards sont les plus nombreux. On peut citer entre autres l'Ordonnance, datée de 1466, de Philippe le Bon sur l'administration et la comptabilité municipale de Lille $^{33}$; un acte établi en 1524 à Senlis pour le tombeau de Guillaume de Montmorency et de sa femme Anne Pot dans l'église Saint-Martin de Montmorency ${ }^{34}$; un contrat de mariage établi en 1695 dans la province de Cambrai ${ }^{35}$; une relation de voyage de Pierre-Louis Jacobs d'Hailly (né en 1669 à Lille), de la fin du XVII' siècle $^{36}$; un autre contrat de mariage dans la province de Cambrai, établi en $1742^{37}$; un arrêt du conseil souverain de Malines, recueilli en $1773^{38}$; le Journal de la Guerre de sept ans (publié en 1796) de Charles Joseph Ligne, né à Bruxelles en $1735^{39}$; les Nouvelles

ordonnant, que sa succession passeroit d'une branche a l'autre, il ne pouvoit qu'entendre une gradation pareille d'une fille a l'autre fait a fait que l'Ainée, ou la ligne descendante de cette Ainée viendroit a manquer » (c'est moi qui souligne).

33 Voir J. Houdoy, « Chapitres de l'histoire de Lille. Le livre Roisin, le privilége de non-confiscation, les comptes de la ville », Mémoires de la Société des sciences, de l'agriculture et des arts de Lille, 1872, p. 174 : « et au regard de ceulx qui sont demourans de la dite rivière du Lys, les III prochains mois passés après un terme escheu, se poura faire exécution du dit terme escheu, et non ainchois, et ainsy de temps en temps et fait à fait, que les dittes rentes escheront, le dit temps durant, sans toutes voies comprendre en ceste surséance les misérables parsonnes non puissans d'atendre, se aucuns en y a. » (c'est moi qui souligne).

34 Voir Anatole de Montaiglon, «Martin Cloistre, de Blois, et Benoist Bonberault, d'Orléans, sculpteurs du XVI ${ }^{e}$ siècle. Histoire du tombeau élevé à Guillaume de Montmorency et à sa femme Anne Pot, dans l'église Saint-Martin de Montmorency », Bibliothèque de l'École des Chartes, 1851, p. 277 : «en quoy faisant ledict seigneur de Montmorancy sera tenu de luy bailler la somme de six cens livres tournois restans, comme dict est, des huit cens livres tournois du marché fait avec ledict deffunt Martin Cloistre, au feur et fait à fait que se feront iceulx ouvrages. »C'est un acte qui commence par « A tous ceulx qui ces présentes lectres verront, Jehan Dole, procureur et conseillier ou bailliage de Senlis, et Jehan de Briquegny, tabellion, gardes des sceaux de la baillie, establiz de par le roy nostre sire en la chastellenye de Senlis, salut. » (p. 274) et qui finit par « Ce fut fait audit Senlis, le vendredy tiers jour de mars, l'an mil cinq cens vingt quatre. » (p. 277).

35 Voir le «Contrat de mariage de Pierre Castrique et de Marguerite-Agnès Cuvelier, le 6 août 1695 », publié dans Alb. Cuvelier-Verley, Généalogie de la famille Cuvelier. 1535-1927, Lille, Société Saint-Augustin, Desclée, de Brouwer, 1927, Société d'Études de la province de Cambrai, Recueil 24, p. 163 : « et fait à fait que lesdits enfans viendront en eage compétent ou prendront estat de mariage ou autre état honnorable » (c'est moi qui souligne).

36 Voir Alcius Ledieu, Voyages en Picardie d'un gentilhomme lillois à la fin du XVII siècle, Cayeux-sur-Mer, 1905, p. 11 : «Le marché au blé et le grand marché sont entourés de grandes galeries en arcades sur lesquelles sont bâties les maisons, toutes d'une même architecture, ce que l'on continue fait à fait que l'on abat les vieilles maisons de bois. » (c'est moi qui souligne).

37 Voir le «Contrat de mariage de Louis de Houchin et d'Antoinette de Béthune, le 20 juillet 1742 », publié par A. Quenson de La Hennerie, dans Société d'Études de la province de Cambrai, Bulletin, 27, 1927, p. 122 : «et ainsi successivement à chaque garçon du d. mariage fait à fait que les aînés viendront à mourir » (c'est moi qui souligne).

38 Voir Arrêts du conseil souverain de Malines, recueillis par M. de Humayn, Lille, Henry, 1773, Arrêt XXXIV, p. 146 : «En cette ville de Malines, le métier de Boucher a divers privilèges ; entr'autres droits il y a quarante haudebanden, dits vieux bancs, affectés aux plus anciens, dans lesquels ceux dudit métier entrent successivement selon l'ordre de leur ancienneté \& serment : ces Bouchers servent à débiter les viandes, \& se donnent quelquefois à louage à ceux qui en sont pourvus. Fait-à-fait que quelqu'un du nombre des quarante vient à faillir, les suivans y entrent selon leur degré \& serment. » (c'est moi qui souligne).

39 Voir Charles Joseph Ligne, Mon Journal de la Guerre de sept ans. Campagne de 1759 et 1760, dans id., Mélanges militaires, littéraires et sentimentales, t. 15, Walter, 1796, p. 186 : «mais il étoit clair que 
démonstrations principales du mécanisme de la vie, publiées en 1861 par François-Auguste Foucard, né à Condé (Nord) en $1800^{40}$.

Quant aux attestations wallonnes, on peut les relever dans les ouvrages suivants : Georges Lentherand (de Mons), de la fin du XV siècle ${ }^{41}$; un édit de 1538 concernant le Duché de Luxembourg et le Comté de Chiny ${ }^{42}$; le Renouvellement des Chartes, Franchises \& Privileges des Férons du Pays \& Comté de Namur, Points \& Status, concernant la conduite \& réglement de leur Style décrété par le Roi [= Philippe, roi de Castille], le 24 Octobre 1635 ${ }^{43}$; un manuscrit autographe de l'Histoire de Mons de Gilles-Joseph de Boussu, né à Mons en $1681^{44}$; l'Hygiène générale (1890), due à Adolphe Burggraeve, né en 1806 à Gand ${ }^{45}$.

l'Aile droit débouchant la premiere, s'appuyeroit d'abord au Katzbach, et couvriroit la Marche de l'Aile gauche qui sous sa protection se formeroit fait-à-fait que les Régimens arriveroient. » (c'est moi qui souligne).

40 Voir François-Auguste Foucard, Nouvelles démonstrations principales du mécanisme de la vie, Lille, Béghin, 1861, p. 29-30 : «Fait à fait que l'azote de l'air est mis en liberté par cette combustion, il s'unit aux principes carbonnés du sang, dont l'excès de carbone vient d'être transformé en acide carbonique, et s'assimile ainsi aux tissus charnus pour les développer [p. 30] ou en réparer les pertes, ainsi qu'à l'albumine du sang, lorsque les aliments n'en procurent pas suffisamment. » (c'est moi qui souligne).

${ }^{41}$ Voir Voyage de Georges Lengherand, mayeur de Mons en Haynaut, à Venise, Rome, Jérusalem, Mont Sinaï \& le Kayre, 1485-1486, Avec introduction, notes, glossaire, \&c. par le marquis de Godefroy Ménilglaise, Mons, Masquillier \& Dequesne, 1861, p. 113: «Et fait à fait que lesdits pélerins descendoyent, ilz passoient entre eulx, et par ung escripvain faisoient escripre le nom de chascun pélerin et le nom de son père. Et fait à fait qu'ilz estoient escrips, l'on les faisoit mener et logier en croustes volsées par gens qu'ilz sembloit qu'ilz les menassent pour cruciffier. » (c'est moi qui souligne).

${ }^{42}$ Voir Edit Touchant les Gaigiéres tenuës de l'Empéreur : Du vingt-troisiéme Juillet 1538, dans Recueil d'édits, ordonnances, declarations et reglemens, concernant le Duché de Luxembourg \& Comté de Chiny, Luxembourg, André Chevalier, 1691, p. $56:$ \& \& Nous pourverrons à la délivrance des deniers que Nous conviendra paier \& débourser, fait à fait que lesdits rachats se feront ; de ce faire vous donnons pouvoir. » (c'est moi qui souligne).

${ }^{43}$ Voir Antoine-Gabriel Jars, Voyages métallurgiques ou recherches et observations Sur les Mines \& Forges de fer, la Fabrication de l'acier, celle du fer-blanc, \& plusieurs mines de charbon de terre, faites depuis l'année 1757 jusques \& compris 1769, en Allemagne, Suéde, Norwege, Angleterre \& Ecosse. Suivies D'un Mémoire sur la circulation de l'air dans les Mines, \& d'une Notice de la Jurisprudence des mines de charbon dans le Pays de Liege, la Province de Limbourg \& le Comté de Namur, Lyon, Gabriel Regnault, 1774, p. 395, article XXII : « Pour faire cesser les difficultés mues à cause des bois, dont lesdits mineurs ont besoin pour lier $\&$ assurer leurs fosses, prétendant que, sans avoir égard aux saisons, \& sans désignations de nos Officiers, sont loisible aux Férons, \& leurs mineurs, de couper bois flexibles indifféremment en nos forêts à l'avenant, \& fait à fait que la nécessité le requiert, pour munir, ceindre \& assurer lesdites fosses. » (c'est moi qui souligne).

${ }^{44}$ Voir Aimé Leroy et Arthur Dinaux, Archives historiques et littéraires du Nord de la France et du midi de la Belgique, t. 2, Valenciennes, 1832, p. 469: «Le 2 d'octobre 1724 les derniers cahiers me furent rendus avec applaudissement, et les premiers fait-à-fait que l'examen et la lecture en fut achevée. » (c'est moi qui souligne).

${ }^{45}$ Voir Adolphe Burggraeve, Hygiène générale comprenant l'hygiène alimentaire, les dyspepsies, l'hygiène des âges, la longévité, Paris, Carré, 1890, p. 172 : « Or les maladies chez le vieillard dérivent de la faiblesse de ses organes, fait à fait que ses forces se paralysent. » (c'est moi qui souligne). 
De Lorraine proviennent les témoignages suivants : un problème proposé par Jean Leurechon, jésuite né en 1591 à Bar le Duc, dans sa Recreation mathematique ${ }^{46}$; un rapport adressé en 1632 à la Chambre des Comptes de Nancy $^{47}$; les Mémoires (publiés en 1665) de François Bassompierre, né en 1579 à Haroué et mort en 1646 à Provins ${ }^{48}$; Les Vraies Constitutions des religieuses, faites par Pierre Fourier et publiées en 1694 à Toul $^{49}$; la Chimie du gout et de l'odorat, publiée en 1755 par Polycarpe Poncelet, né à Verdun vers $1720^{50}$; la Théorie des machines publiée en 1784 par Sébastien Maillard, né à Lunéville en $1747^{51}$; une lettre de 1793 de Jean Nicolas Houchard, né en 1738 à Forbach $^{52}$; un article sur le hunage ou la holmée que Léon Germain de Maidy a publié en $1923^{53}$.

${ }^{46}$ Voir Jean Leurechon, Recreation mathematique, composée de plusieurs problemes plaisans et facetieux en faict d'Arithmetique, Geometrie, Mechanique, Optique, \& autres parties de ces belles sciences, $4^{\mathrm{e}}$ édition, Paris, Boutonné, 1627, $75^{\mathrm{e}}$ problème « Des Aeolipiles, ou boules à souffler le feu ", p. 129-130 : «D'autres se contentent d'un simple tuyau dressé à plomb, un peu evasé par le haut, pour y mettre une petite boule qui sautelle par des-[p. 130]-sus, fait à fait que les vapeurs sont poussées dehors. » (c'est moi qui souligne).

47 Voir le «Rapport adressé à la Chambre, le 22 août 1632, par les commissaires qu'elle avait délégués pour informer au sujet d'une requête par laquelle les habitants de Frouard demandaient à être exempts, pour six années, de l'aide Saint-Rémy et des autres rentes auxquelles ils étaient attenus », dans Henri Lepage, Les Communes de la Meurthe. Journal historique des villes, bourgs, villages, hameaux et censes de ce département, t. 1, Nancy, Lepage, 1853, p. 390b: «en sorte qu'au lieu d'y pouvoir vaquer, ils auraient été contraints d'envoyer leursdits chevaux aux champs fait à fait que les herbes croissaient » (c'est moi qui souligne).

${ }^{48}$ Voir Journal de ma vie. Mémoires du maréchal de Bassompierre. Première édition conforme au manuscrit original publiée avec fragments inédits pour la Société de l'Histoire de France par le Marquis de Chantérac, t. 2, Paris, Renouard, 1873, p. 368 (novembre 1621) : "Apres j'ostay les mousquetaires desdites traverses et places fait a fait que je n'en avois plus de besoin »; p. 372 (id.) : «et fait a fait que l'on destachoit un batteau, je le faisois descendre à val. » (c'est moi qui souligne).

${ }^{49}$ Voir Les Vraies Constitutions des religieuses de la congregation de Nostre Dame Faites par le Venerable serviteur de Dieu Pierre Fourier leur Instituteur, \& General des Chanoines reguliers de la Congregation de nôtre Sauveur, approuvées par nôtre Saint Pere le Pape Innocent $\mathrm{X}, 2^{\mathrm{e}}$ édition, Toul, Laurent, 1694, p. 54 : «En toutes les classes, fait à fait que des écolieres auront achevé de dire leur leçon, la Maîtresse qui les recorde leur montrera dans leur livre ce qu'elles devront étudier pour la leçon suivante. » (c'est moi qui souligne).

${ }_{50}$ Voir Polycarpe Poncelet, Chimie du gout et de l'odorat ou principes Pour composer facilement, \& à peu de frais, les Liqueurs à boire, \& les Eaux de senteurs, Paris, Le Mercier, 1755, p. 223 : « Fait à fait que vous les retirerez du blanchissage, vous les étendrez sur un clayon ou sur un linge blanc, afin qu'elles puissent jetter toute leur eau » (c'est moi qui souligne). Sur l'auteur, voir Frédéric Charbonneau, L'art d'écrire la science. Anthologie de textes savants du XVIII siècle français, Saint-Nicolas (Québec), Les Presses de l'Université Laval, 2005, p. 127.

${ }^{51}$ Voir Sébastien Maillard, Théorie des machines mues par la force de la vapeur de l'eau, Vienne et Strasbourg, Gay, 1784, p. 112 : «Comme fait à fait que l'on s'éloigne du niveau de la mer le terrain va ordinairement en s'élevant, \& le mercure en baissant dans le baromètre, ce qui montre que le poids de la colonne d'air diminue ; on voit, sur-tout dans les pays très-élevés, qu'il est de la dernière conséquence de connoître le poids de la colonne d'air qui répond à l'endroit où la machine doit s'établir. » (c'est moi qui souligne). Sur l'auteur, voir Alain Petiot, Au service des Habsbourg. Officiers, ingénieurs, savants et artistes lorrains en Autriche, Paris, Messene, 2000, p. 58.

52 Voir sa lettre au ministre de la guerre, le 29 septembre 1793, dans Correspondance générale de Carnot publiée avec des notes historiques et biographiques par Etienne Charavay, t. 3, Paris, Imprimerie 
Les témoignages champenois sont plutôt rares ; ils se trouvent dans un journal anonyme de $1775^{54}$, une observation d'I. Salesse datée de $1894^{55}$, et une remarque d'Émile Guénard dans son patois de Courtisols de $1905^{56}$.

Ainsi, la locution fait à fait que n'est pas seulement picarde comme le disaient Charles Athanase Walckenaer, Henri Régnier, Georges Couton et Marc Fumaroli. Elle n'est pas non plus limitée à la Champagne comme le voulaient Raymond Josse et Jean-Pierre Collinet. On ne peut pas non plus dire qu'elle est picarde et champenoise en se basant sur une autre note de Walckenaer. Il est également difficile de suivre Walther von Wartburg qui croyait qu'elle n'est attestée qu'au seul XVII siècle. Elle a en fait une histoire assez longue, depuis le milieu du $\mathrm{XV}^{\mathrm{e}}$ siècle jusqu'au début du $\mathrm{XX}^{\mathrm{e}}$ siècle, et une aire de diffusion assez large, qui couvre les domaines picard, wallon, lorrain et champenois. L'information ainsi obtenue servira à étoffer la note sur le vers $11 \mathrm{du}$ Coche et la Mouche et à compléter l'article factum du FEW. D'autres régionalismes de La Fontaine ${ }^{57}$ mériteront aussi d'être examinés de près.

\footnotetext{
Nationale, 1897, p. 229 : «Fait à fait que j'avançais et que j'aurais battu tous les corps détachés des ennemis, je me grossissais et j'arrivais sur Cobourg avec 70000 hommes » (c'est moi qui souligne).

${ }^{53}$ Voir Léon Germain de Maidy, « Note sur une ancienne coutume populaire. Le hunage ou la holmée », dans Le Pays lorrain et le pays messin. Revue mensuelle illustrée. Littérature, Beaux-Arts, Histoire, Traditions populaires, 15, 1923, p. 127: «Le hûnage est une coutume singulière et peu louable : les jeunes garçons guettent les filles dans les rues; fait à fait que l'une se laisse saisir, deux d'entre ceux la prennent par les épaules et par les pieds, puis la balancent, tandis qu'un autre ou plusieurs passent au-dessous. » (c'est moi qui souligne).

${ }^{54}$ Voir Journal anonyme du XVIII siècle, dans Journal de Dom Pierre Chastelain, Bénédiction Rémois 1709-1782 Avec ses Remarques sur la température et la vigne suivies d'un autre Journal et d'Observations analogues jusqu'en 1848 Publiés sur les documents originaux de la Bibliothèque de Reims avec une introduction et des notes par Henri Jadart, Reims, Michaud, 1902, p. 276 : « La farine s'est vendue 14 livres, 13, 12,11, 10 livres le septier, c'est à dire qu'au lieu d'augmenter, elle a baissé fait à fait que la moisson a approché, et le froment à peu près de même. » (c'est moi qui souligne).

55 Voir I. Salesse, Un coin de la Champagne et du Valois au XVII siècle. Jean de La Fontaine - Marie Héricart, Château-Thierry, Lacroix, 1894, p. 85 : «Parmi les locutions empruntées par La Fontaine au dictionnaire de Château-Thierry et corrigées très probablement ensuite sur les conseils de Marie Héricart, on peut citer la suivante, tirée de la fable "Le Coche et la Mouche" édition de 1671. Fait à fait que le char chemine, etc... pour : à mesure que, etc... Cette locution est encore en usage à Château-Thierry et dans les environs. » (c'est l'auteur qui souligne).

56 Voir Émile Guénard, Le patois de Courtisols, ses rapports avec les patois marnais, Châlons-sur-Marne, Imprimerie de 1'Union Républicaine, 1905, p. 159 : « Fait à fait, loc. adv. ; - tout doucement, au fur et à mesure (Court. et toute la Marne). Fait à fait que, loc. conj., à mesure que : "Fait à fait que le char chemine", La Font., Fabl. VII, 9, var. - Cf. le pat. fât, fait (Perthois). ». (c'est l'auteur qui souligne).

57 Je pense par exemple au syntagme le chef d'octobre au sens du «premier octobre » et au substantif chuchillement qui signifie « chuchotement ».
} 